\title{
System of Systems Simulation Driven Wildfire Fighting Aircraft Design and Fleet Assessment
}

\author{
Prajwal Shiva Prakasha ${ }^{1}$, San Kilkis ${ }^{2}$, Nabih Naeem ${ }^{3}$, Patrick Ratei ${ }^{4}$, and Björn Nagel ${ }^{5}$ \\ German Aerospace Center (DLR), Hamburg, 21129, Germany
}

\begin{abstract}
Large wildfires are increasingly occurring phenomenon in several since the past few years. The suppression of wildfires is complex considering heterogeneous independent constituent systems operating together to monitor, mitigate, and suppress the fire. In addition, the management of the disaster response involve multiple institutions in collaboration. Recognition of this wildfire fighting scenario, as a System of Systems (SoS) is valid. Aerial vehicles may play a big role in firefighting considering monitoring and suppression at early stages when the fire is still small. Thus, there is scope for designing a new Unmanned Aerial Vehicle (UAV) with a payload of $250 \mathrm{~kg}$ to $500 \mathrm{~kg}$ for aerial forest fire suppression, using a SoS wildfire simulation driven aircraft design approach, where the individual optimum performance of a system, especially of a new aircraft for firefighting, does not guarantee optimum overall firefighting mission effectiveness. Whereas an optimum combination of fleet, technology and operational tactics can effectively suppress fire. For this reason, this research focuses on four different aspects:

1) Applying the inverse design paradigm to a wildfire suppression air vehicle by coupling a fire propagation cellular automata model with a stochastic agent-based simulation of an evolved firefighting SoS. An efficient SoS framework to Evaluate fleet performance.

2) Four System of systems - system - subsystem interlinking research questions are addressed with corresponding sensitivity results. The impact of wildfire based on vehicle fleet size, vehicle architecture (Tiltrotor, Compound Heli, Multirotor or Lift cruise), payload carrying capability, response time and cruise speed.

3) The evolution of perfect combination of aerial vehicle fleet with different vehicle architectures, technologies and performances using simulations.

4) Obtaining a set of system level (aircraft level) Measures of Performance (MoP) for the large suppression UAVs that produce improved SoS-level Measures of Effectiveness (MoE) during an initial attack quantified by containment time and total fire burnt area. As addressed by research questions and results. The response time and Number of Aircraft has large impact on success of the firefighting mission. As the time advantage deteriorate, the wild fire expands exponentially.
\end{abstract}

\section{Introduction}

As the northern part of the earth warms at a faster pace, the unusual heat is causing forests to dry out, leading to an unprecedented severity of wildfires. In Europe alone, the area susceptible to fire is projected to increase $200 \%$ by the end of the 21st century [1]. Already the intense forest-fires in Sweden during July of 2018 prompted the activation of the European Union Civil Protection Mechanism which constituted the largest forest fire operation of the last decade in Europe, where 80 vehicles and 360 personnel were deployed from seven countries [2]. Therefore, the positive emergent ability to contain such wildfires is possible through the collaboration of various operationally independent and useful heterogeneous systems that are geographically distributed. These systems include ground, air, and space vehicles, ranging from bulldozers and fire engines, to helicopters, fire monitoring air vehicles, and earth observation satellites. Often these vast systems are operated by separate agencies and are therefore managerially independent and evolve their capabilities on their own.

Thus, recognition of this firefighting scenario, a complex system, as a System of Systems (SoS) is valid according the Maier criteria [3]. Thus, the scope of designing a new air vehicle for fire suppression, using a System

\footnotetext{
${ }^{1}$ Research Scientist, Institute of System Architectures in Aeronautics. AIAA Member.

${ }^{2}$ Research Student, Institute of System Architectures in Aeronautics.

${ }^{3}$ Research Student, Institute of System Architectures in Aeronautics.

${ }^{4}$ Research Student, Institute of System Architectures in Aeronautics.

${ }^{5}$ Founding Director and Head of Institute, Institute of System Architectures in Aeronautics.
} 
of Systems oriented flight vehicle design approach, where the individual optimum performance of a system, especially of a new aircraft for firefighting, does not guarantee optimum mission effectiveness.

The use of autonomous vehicles for combating wildfires is not a new avenue of research; however, compared to using Unmanned Aerial Vehicles (UAVs) for fire detection and fire-front monitoring, [4] concluded that the published research on using UAVs for fire suppression is scarce and lacks detail. Furthermore, according to [5] much work remains to make the use of UAVs affordable and technically viable for firefighting. Even rarer is the analysis of suppression UAVs from a SoS perspective. The usefulness of UAVs during extended attack scenarios, necessitating the use of ground assets, requires further research. In fact, a significant increase in the probability of successful containment is observed when aircraft and ground assets collaborate [6], since aircraft enhance the effectiveness and efficiency of ground suppression forces and make fire-line construction safer and easier by decreasing the fire intensity and slowing fire-front propagation [7]. Therefore, this research will serve to fill this gap in present literature by simulating a larger-scale SoS comprised additionally of ground units and legacy manned aircraft that execute direct, parallel, and indirect attack strategies dynamically. In doing so, this research can quantify effectiveness in worst-case wildfire scenarios where extended attack is necessary.

In order to structure the SoS design, a framework is developed to establish the key variables of the design, construct the SoS model, execute the simulations, and analyze the output data to constrain the design space. The concept of operations of current firefighting and tactics, as described by firefighting handbooks, is implemented in the Multi-Agent Based Simulation (MABS) with a rule-based approach where properties of the fire-model trigger predefined containment and suppression actions. Fig. 1 depicts a rule-based suppression strategy, where the incident commander dynamically assigns assets and firefighting strategies to specific fire-fronts based on visibility and intensity. Fig. 2 shows an envisioned coupling between the discrete cellular space and the continuous operational space in which the firefighting agents reside and interact. Finally, as it is important to represent uncertainty in a SoS, stochasticity will be incorporated into the simulation through use of probability distributions for variables such the start location and time of a fire, as well as environmental conditions such as wind and ambient temperature.

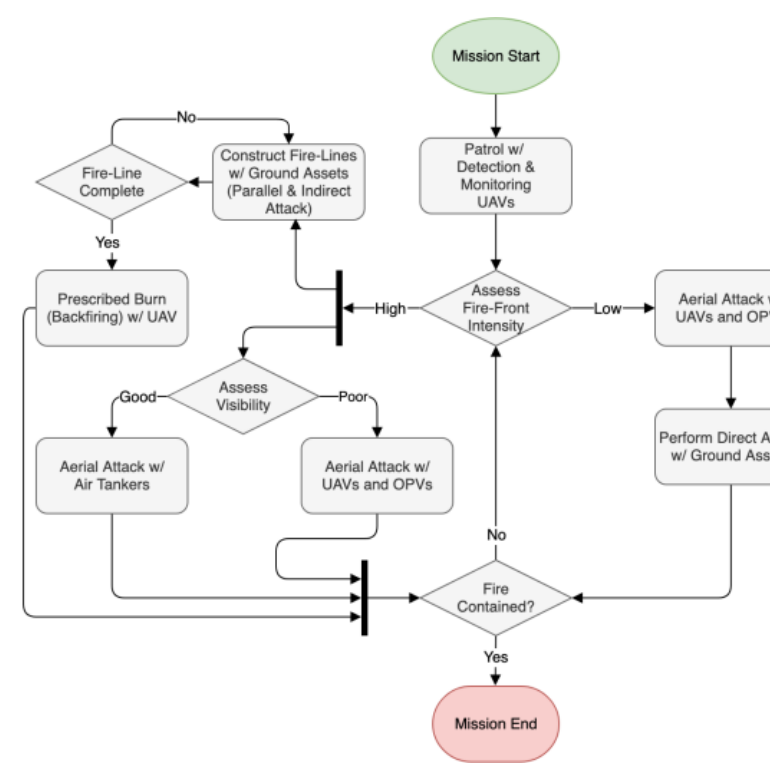

Fig. 1 Proposed SoS wildfire suppression strategy

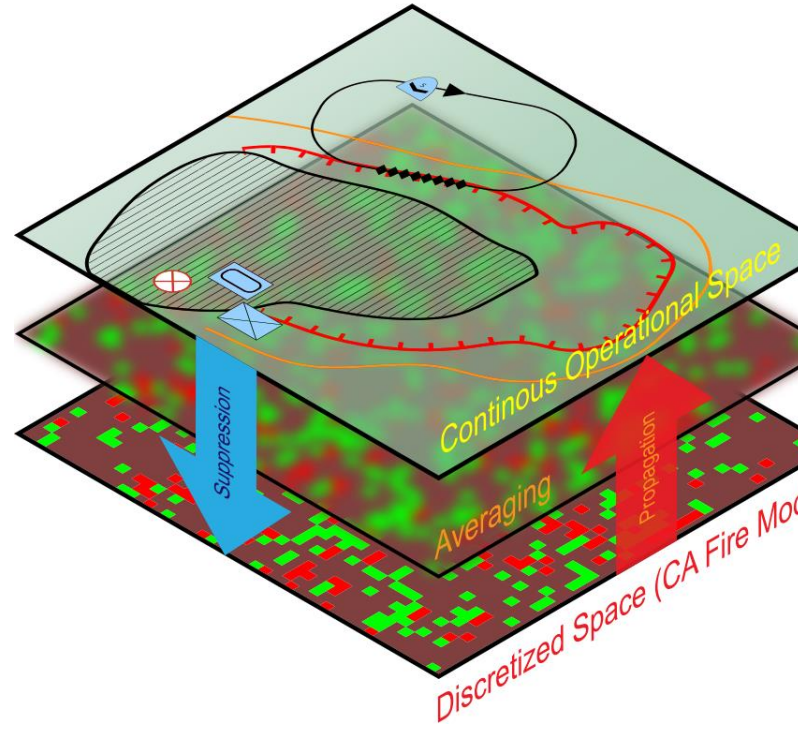

Fig. 2 Coupling of fire model and firefighting agents

Once a coupling between the fire-model and MABS is established, the Overall Effectiveness Criterion (OEC) will be used to assess the MoEs of the firefighting mission. For this research, the OEC of containment time and burnt area is used, which are typical indicators of success [8]. 


\section{System of System Framework}

The following Fig. 3 provides the implementation of fire fighting vehicle design where the simulation drives the design process. As per Fig. 3 based on multiple scenario of wildfire; Efficient Vehicle configuration architecture need to be design for with best range, quick turnaround, low altitude terrain hugging flying quality, speed, water retrieval and water dropping subsystem. Multiple heterogeneous fleet of vehicles (suppression fighting and suppression super tankers, small rapid response vehicles) need to be designed for optimal combined operations. The simulation acts as the testbed on which different homogenous and heterogenous fleets can be analyzed and evaluated. As a simple example of what the simulation could help answer: the question of whether a large fleet of small agile aircraft or a small fleet of large aircraft would perform better at wildfire suppression. Moreover, simulations can help find the ideal balance between the multiple architectures within a fleet and help in driving the design of the vehicle or fleet.

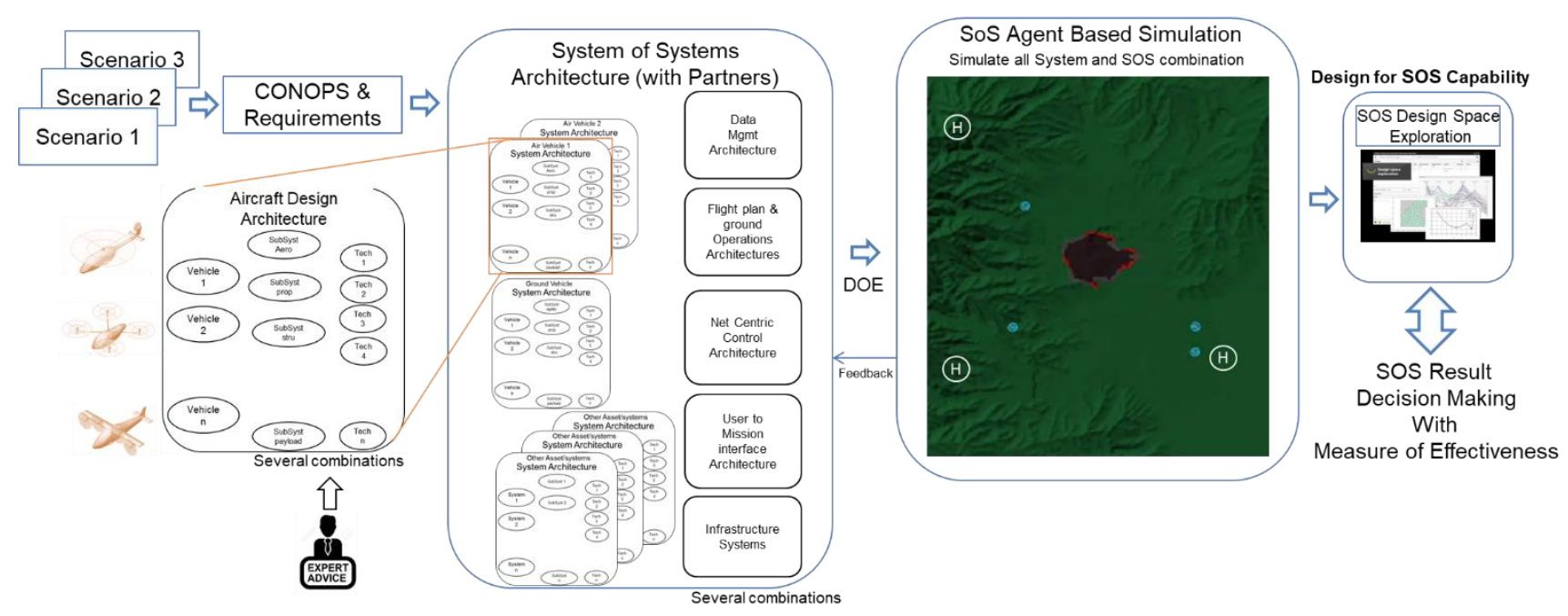

Fig. 3 Simulation embedded System of System aircraft design framework

\section{A. Aircraft Design and Operations}

The aircraft design as first approach is evolved from the conceptual eVTOL aircraft design methodology by Brown and Harris [12]. Multirotor, compound helicopter, lift + cruise, and tiltrotor configurations are designed with their respective performance characteristics. This is a simplistic preliminary study to propagate the design performance of one vehicle to fleet level and to achieve the proof of concept for the purpose of designing UAVs for wildfire fighting. The underlying method utilizes momentum theory for hover segments and simple steady flight equations for cruise segments. It allows for investigation of various eVTOL aircraft configurations. The design method will be constantly evolved and updated in subsequent publications, in fact strides have been made parallel to this study in [Cite SSOS paper] to improve the aircraft design methodology. Beyond configuration effects of propulsion, weights, and aerodynamics, the aircraft technology assumes a battery pack specific energy of $300 \mathrm{Wh} / \mathrm{kg}$ with a usable energy fraction of 0.8 . The aircraft are sized for a reserve loiter of 20 minutes for contingencies, which probably would not be required for unmanned vehicles operated in non-urban environments. The aircraft are expected to have autonomous operating capabilities with remote intervention if required. The mission profiles for the sizing process are provided in Fig. 4. For the same profile, a short-range sizing mission $(30 \mathrm{~km})$ and a mid-range sizing mission $(60 \mathrm{~km})$ are considered, varying only the cruise range. In Fig. 5, two possible simulation mission profiles are presented. To the left a single suppression mission and to the right an extended suppression mission with resupplying. Furthermore, two payload masses, $250 \mathrm{~kg}$ and $500 \mathrm{~kg}$, are considered.

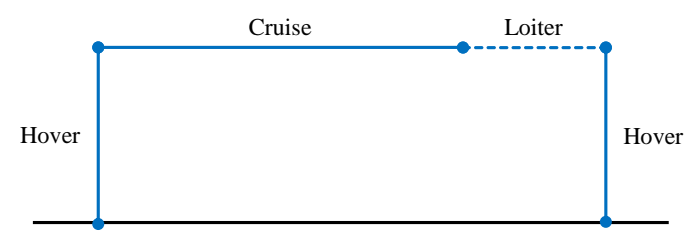

Fig. 4 Sizing mission profile 


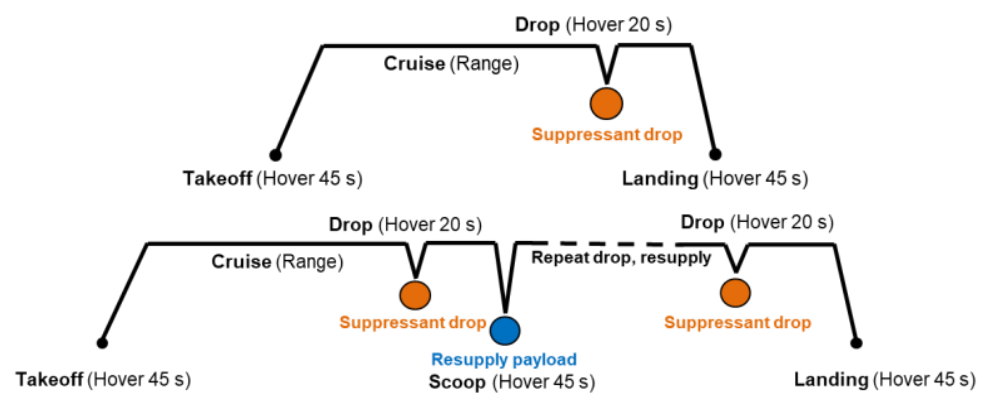

Fig. 5 Simulation mission profile: (Left) Single suppression drop, (Right) Extended with resupply and further suppression drops

\section{B. Multi Agent-Based Simulation}

While the MABS for the wildfire fighting case study will be explained in the following, the detailed work on the underlying simulation toolkit is extensively described in a separate paper presented at this conference [13].

The simulation model has four major components, which will be explained in the following. The components are namely:

1) Wildfire model

2) Multi agent-based simulation

3) Wildfire suppressant drop model

4) Aircraft performance and energy model

\section{Wildfire Model}

The simulations advancement in this research is to incorporate the medium-fidelity Cellular Automata (CA) wildfire spread model of [9], as depicted in Fig 6Error! Reference source not found.. This CA-based wildfire spread model was selected due to its ability to model the influence of combustibles, temperature, humidity, wind, and terrain in physical time steps. Its use of physical time-steps is important when coupling it with the firefighting agents. Furthermore, the ability to model temperature, humidity, and wind are paramount to representing the reduced intensity of wildfires at night.

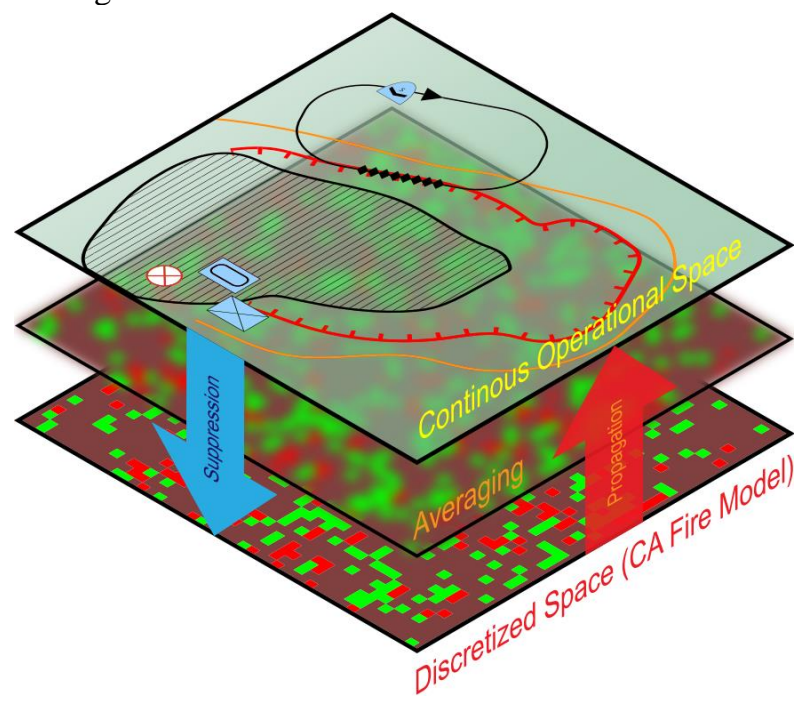

Fig. 6 Wildfire simulation model

The basis of any CA model is the processing of information in surrounding cells in order to evaluate so-called transition rules. Formally, each cell within a CA model is represented by a set of cell-states described by

Table 1. 
Table 1 Specification of cellular automata fire states

\begin{tabular}{|c|c|c|c|}
\hline State Name & Symbol & Color & Description \\
\hline Non-Flammable & $S_{0}$ & $\square$ & A cell with no fuels (i.e. water, roads, rock, etc.) \\
\hline Combustible & $S_{1}$ & $\square$ & $\begin{array}{l}\text { A cell that has fuel content and can ignite (i.e. farmland, forest, houses, } \\
\text { etc.) }\end{array}$ \\
\hline Early Burning & $S_{2}$ & $\square$ & $\begin{array}{l}\text { A cell that has ignited. If a cell transitions to early burning, then it will } \\
\text { automatically transition to a full burning state at the next iteration. }\end{array}$ \\
\hline Full Burning & $S_{3}$ & $\square$ & $\begin{array}{l}\text { A cell that is fully ignited which has the capability to ignite neighboring } \\
\text { cells. If all neighboring cells are either full burning, beyond the full } \\
\text { burning state, or are non-flammable, then the cell will start } \\
\text { extinguishing in the next iteration. }\end{array}$ \\
\hline Extinguishing & $S_{4}$ & $\square$ & $\begin{array}{l}\text { A cell in which the fire is rapidly losing intensity. If a cell has } \\
\text { transitioned to this state, then it will be burnt in the next iteration. }\end{array}$ \\
\hline Burnt & $S_{5}$ & $\square$ & $\begin{array}{l}\text { A cell in which a fire has fully extinguished. It is assumed that these } \\
\text { cells cannot be re-ignited as their fuel content is now zero. }\end{array}$ \\
\hline
\end{tabular}

As shown by Fig. 7, the state of each cell depends on the values of its 8 neighboring cells, which constitutes a Moore neighborhood. As the simulation time advances with a discrete time step, $\Delta t$, the cells which are burning ignite neighboring cells with a rate of spread equal to the computed local spread rate, $R$. Computationally, as each cell in the array performs the same calculations and logical operations it is identical to convolution kernels used in image processing which are also called Sliding Window Algorithms (SWOs) and allows for massive parallelization. Due to how inverse design requires thousands of simulation runs, it is of vital importance to utilize this opportunity to reduce runtime. However, the performance of implementing the fire-spread model with Python objects would not have been acceptable due the high computational overhead spent on querying dynamic types. To avoid these traditional shortcomings of a dynamically typed language, the Numba package was used, which is a Just-in-Time (JIT) compiler that translates pure Python code into efficient machine code. Additionally, the parallelization functionalities of Numba enabled the wildfire spread model to target multi-core CPUs or GPUs as well as utilize the same device functions on both CPUs and GPUs, increasing code commonality.

Unlike CPUs which handle data caching and control-flow much better, GPUs are built for data processing and are invaluable when the ratio of computations performed per data access is high. However, unfortunately GPU programming has a steep learning curve and poses challenges for novices such as increased complexity with managing different types of device memory [10]. Therefore, programming an efficient algorithm on the GPU requires a good understanding of the underlying architecture of the device. This also means that algorithms are rather architecture dependent and cannot be ported to the GPUs of different manufacturers easily. However, regardless of the challenges, due to the highly repetitive and independent nature of Sliding Window Algorithms (SWOs), utilizing GPUs for this task has been gaining popularity in research [10]. 


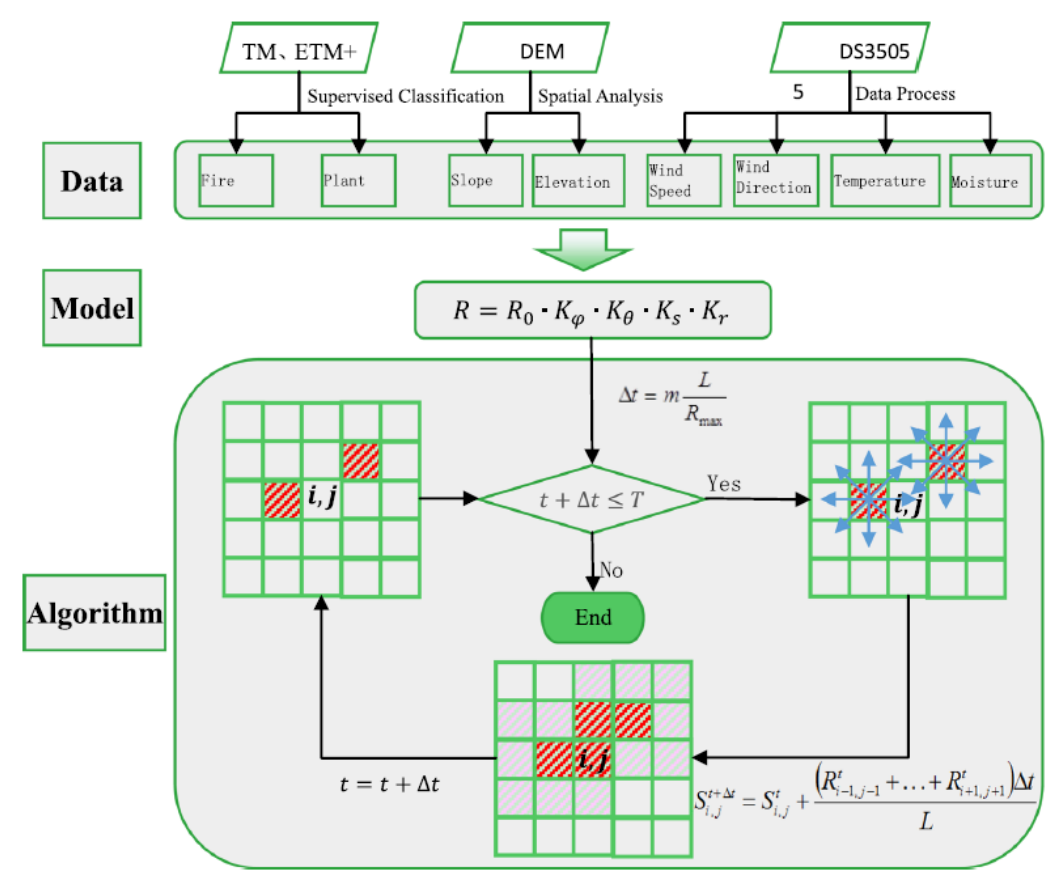

Fig. 7 Overview of Wildfire Spread Model

\section{Multi-Agent Based Modeling}

Each fire fighting vehicle is modeled as an agent the performance characteristics are embedded into the agent while simulations are performed (see Fig. 8).

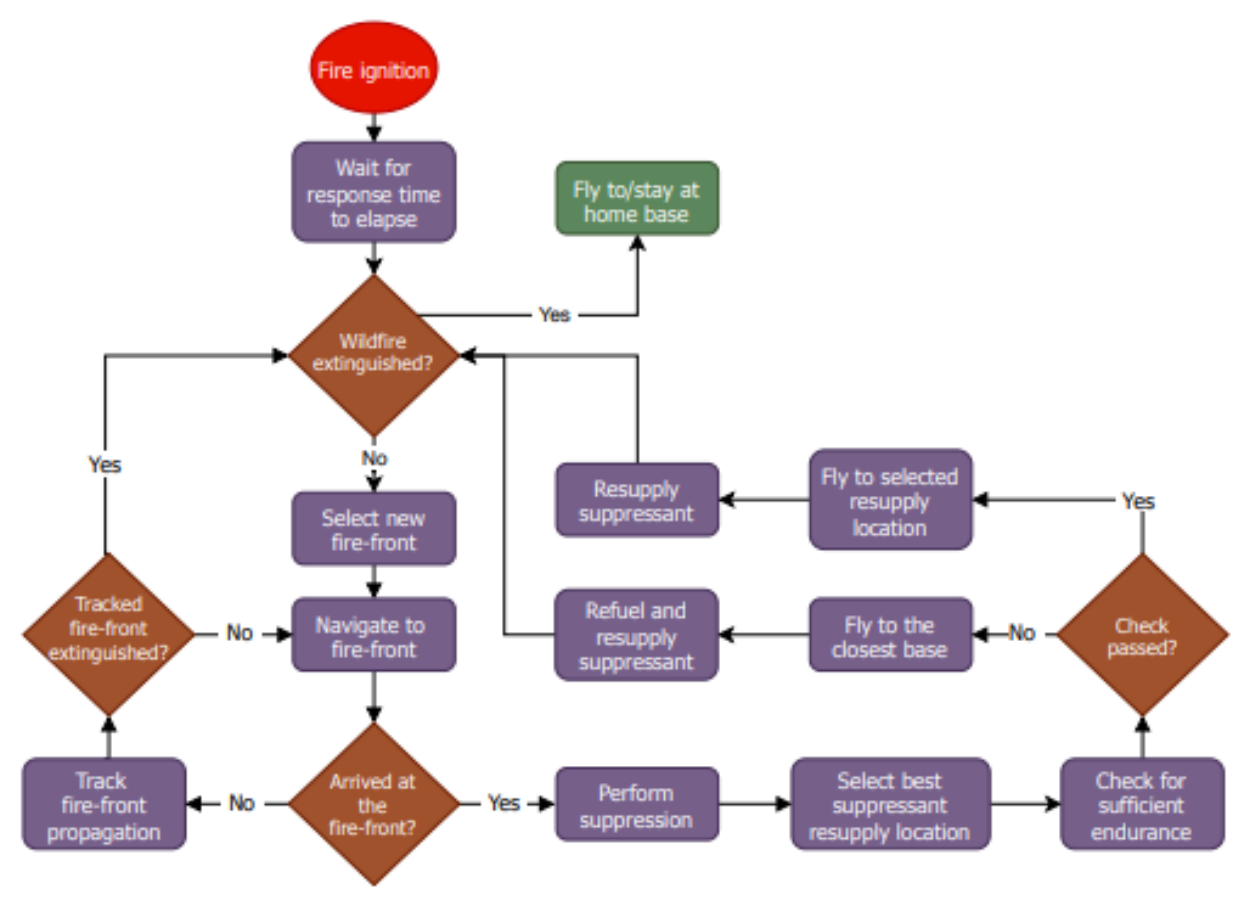

Fig. 8 Logic for Suppression UAV Agents

\section{Wildfire Suppressant Drop Model}

Initially the suppression patch dimensions were kept constant for all firefighting UAVs, regardless of their payload bearing capacity or any other variable. Thus, it was necessary to develop a low to medium-fidelity suppression patch model that could connect the aircraft parameters to suppression effectiveness. The main goal was to find a relation determining the suppression patch dimensions from aircraft payload mass and at most two 
other input parameters. The suppressant drop experiment data, which was collected from [11], was obtained from a wide variety of aircraft types and delivery systems. In general, the delivery systems in the study can be first classified (as Model) into fixed-wing aircraft and rotorcraft. Next, the fixed wing aircraft delivery systems can be further divided into gravity deployment systems and pressurized systems, while the rotorcraft can be further split into the ones utilizing a so-called "helibucket" and the ones using a fixed tank. This variety makes the data well suited for the SoS case study suppression model, since the model has to be able to predict the suppression patch dimensions for a large range of simulated firefighting aircraft. The data in [11] was generated by USDA Forest Service during real-scale water drop tests for different delivery systems. In total, 20 different aircraft types were included in the experiments. Some aircraft performed multiple runs and produced multiple data points while others only performed a single drop which resulted in a single data point for that type. The measurements were conducted by simply arranging cups in a grid pattern over the ground and collecting the water released from the aircraft. Next, the cups were closed and weighed. The results obtained gave information about the dimensions of the suppression patch, but also about the distribution of water over this patch. A top-down visualization of the drop patterns of 3 different aircraft from the data-set can be seen in Fig. 9. Examples of the water drop distribution contours from 3 different aircraft, flying left to right. Top: BV-107 helicopter using the 1,000-gallon (3,785 L) Griffith helibucket. Middle: CDF S-2T Turbo Tracker with 1,200-gallon (4,542 L) constant-flow tank. Bottom: Evergreen Boeing 747 with 18, 000-gallon $(68,137 \mathrm{~L})$ pressurized tank. [11]
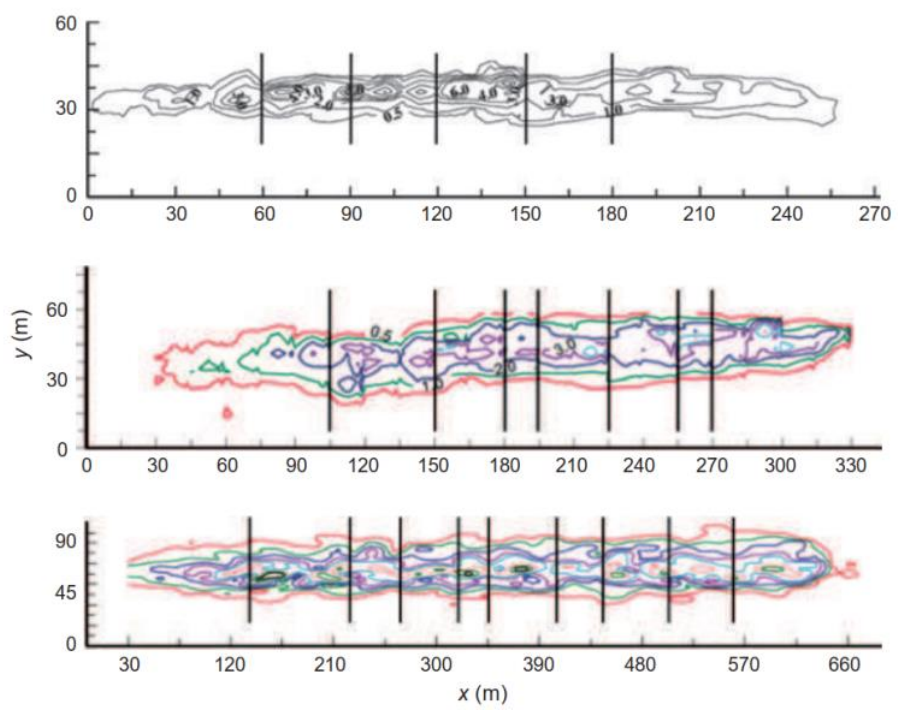

Fig. 9 Examples of the water drop distribution

First, when multiple data points were obtained by USDA Forest Services for the same aircraft type only the most extreme values were presented in [11]. Therefore, the average values between the minimum and maximum values given were considered for this suppression model. Second, upon further examination of the data it was decided to ignore the data for Evergreen Boeing 747 using a pressurized jet delivery system. The aircraft was deemed too large and the delivery system too sophisticated to be a valid representation of the small to medium size UAVs the wildfire case study is meant to simulate. Thirdly, since the total suppression patch area was not directly available from the data set an assumption had to be made to be able to obtain an approximate area from length and width. Based on Fig. 9 it was assumed that all suppression patches can be approximated by an ellipse with length and width of the patch corresponding to the major and minor axes of the ellipse. Finally, different data set parameters were plotted in an effort to discover the strongest correlations between aircraft or environmental variables, and the resulting suppression patch dimensions. As expected, the strongest correlation between the aircraft payload volume and dimensions of the suppression patch was observed in the total area of the suppression patch. The Fig. 10 displays this correlation for different types of aircraft and suppressant delivery systems. It can be observed that the $\mathrm{R} 2$ value of the linear regression for all data combined is around 0.49 , which was deemed adequate for the expected fidelity of the model. Looking at the fixed-wing aircraft subset, however, reveals that there is effectively no correlation whatsoever. Thus, it is paramount to update the model once the aircraft type is considered in the wildfire case study. 

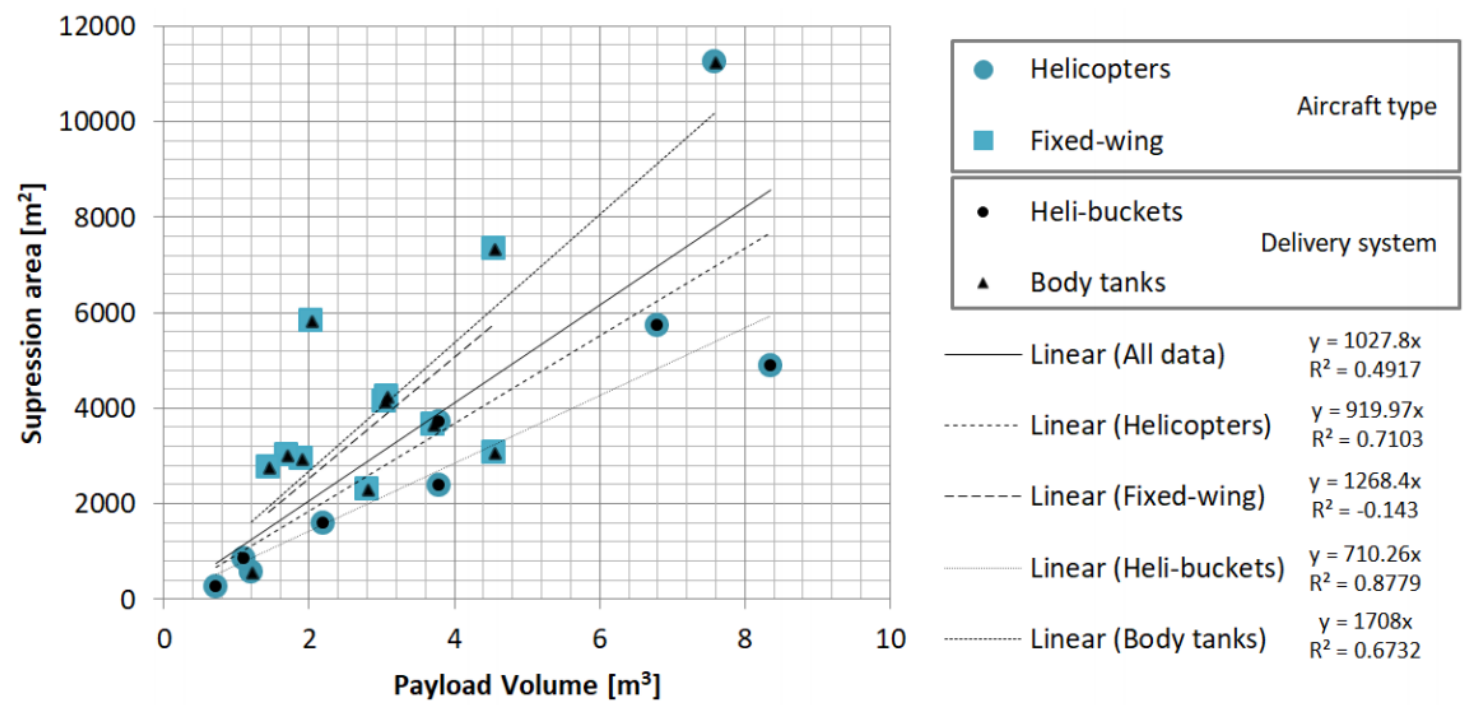

$\begin{array}{ll} & y=1268.4 x \\ R^{2}=-0.143\end{array}$

Linear (Heli-buckets) $\begin{aligned} & y=710.26 x \\ & R^{2}=0.8779\end{aligned}$

10

$y=1708 x$
$R^{2}=0.6732$

Fig. 10 Drop pattern area versus the aircraft suppressant payload volume (assuming elliptic suppression patch).

Fig. 11 shows the suppressant flow rate and the resulting patch width. This plot is presented because the depicted variables exhibit the strongest correlation of any parameters in the data-set and because they were subsequently selected to be a part of the model. Due to the large number of variables influencing the patch width and the lack of sufficient research into the topic it was hard to predict what mathematical function type would best describe the given correlation in reality. The power function regression was therefore selected mainly due to the highest R2

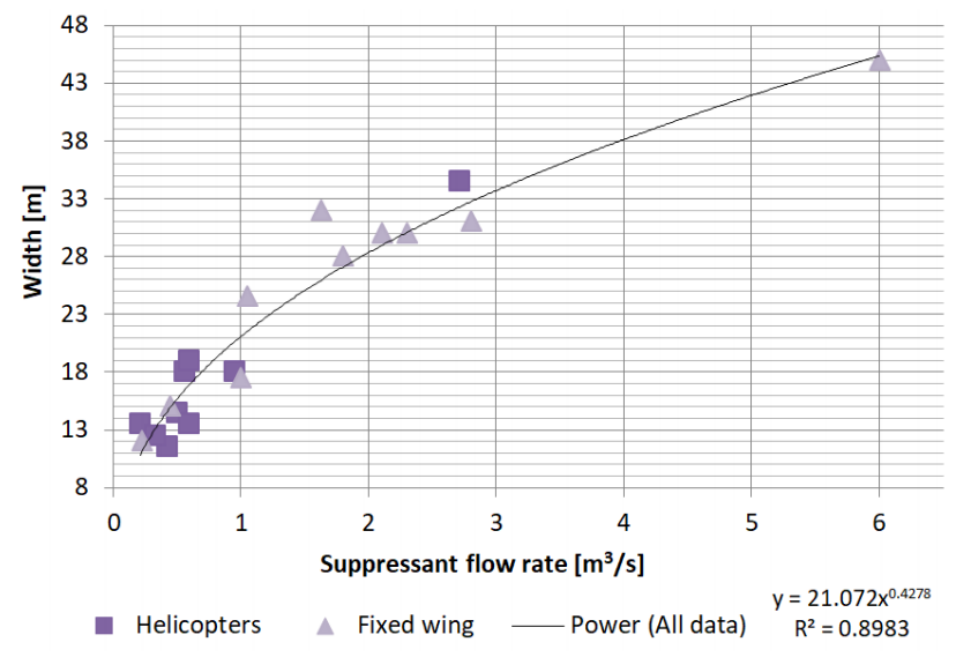

Fig. 11 Drop pattern width versus the aircraft suppressant payload flow rate

The size of a suppressed area caused by a single suppressant drop can vary significantly based on a plethora of parameters besides the more obvious ones, such as payload volume, aircraft ground speed and suppressant flow rate. Deployment technology, fire intensity, drop height, and wind are just some of the other major factors influencing the suppression patch dimensions. However, even predicting a select few of these major influences within the wildfire case study would result in an unwanted increase in the number of simulation input variables. Thus, many assumptions had to be made to produce a uniform model based on a limited number of variables.

Following the drop pattern data analysis these assumptions were deemed admissible to simplify the model to an acceptable level:

- The suppression patch is always elliptical

- The suppressant payload volume fully determines the resulting suppression patch area

- The suppressant flow rate fully determines the suppression patch width

- Any fire within the drop patch will be fully suppressed 
- The aircraft ground velocity, wind, release door size, drop height, deployment technology, terrain slope and any other minor effects on the suppression are neglected

The final model implements the payload volume versus suppression area linear regression seen in Equation 1 from Fig. 10. Assuming water as the suppressant fluid, the model estimates that each kilogram of payload covers around a square meter of ground area. Next, the minor axis of the elliptical suppression patch is obtained with Equation 2 following from the power-function regression curve of the Fig. 11:

$$
\begin{gathered}
A_{\text {supress }}\left[\mathrm{m}^{2}\right]=1028 V_{\text {payload }}\left[\mathrm{m}^{3}\right] \\
B_{\text {supress }}\left[\mathrm{m}^{2}\right]=21.07 Q\left[\frac{\mathrm{m}^{3}}{\mathrm{~s}}\right]^{0.428}
\end{gathered}
$$

The major axis of the patch than simply follows from rearranging the equation for the area of an ellipse:

$$
A_{\text {supress }}=\frac{4}{\pi} \frac{A_{\text {supress }}}{B_{\text {supress }}}
$$

To translate the suppression model from continuous space to the rasterized CA grid of the SoSID wildfire example fire model additional steps were required. The issue and the solution will be demonstrated using an example. Assume a CA grid with a resolution of $10 \mathrm{~m}$ and the suppression model gives a suppression patch length of $61 \mathrm{~m}$. Because of the resolution of the grid, the patch can either be mapped onto the grid as $60 \mathrm{~m}$ or $70 \mathrm{~m}(6$ or 7 cells). The instinctive solution would be to simply round the length to the nearest full cell, in this case $60 \mathrm{~m}$. Now assume the payload has increased and the new length is $64 \mathrm{~m}$. Again, the value is rounded down to $60 \mathrm{~m}$, making the higher payload aircraft no more effective at suppressing the fire. To overcome this a probability function that determines whether the parameter should be rounded up or down was added to the model. Returning back to the first example, a length of $61 \mathrm{~m}$ would have a $10 \%$ chance of being rounded up to $70 \mathrm{~m}$ and a $90 \%$ chance of being rounded down to the $60 \mathrm{~m}$. Consequently, even a slight change in payload mass has a proportional effect once enough suppressions are performed in a simulation.

\section{Aircraft Performance and Energy Model}

The simulation framework employs an energy model evaluating the performance computations of the aircraft. The maximum battery energy of the aircraft is reduced by the usable energy fraction and the energy required for the reserve mission. Power consumption data are provided to the simulation by the sizing tool for full payload and no payload states. The available energy of the aircraft is updated in every iteration considering its flight state and payload. As this study is in the conceptual aircraft design level, simplifying assumptions are taken in the mission profile related performance, as such the exact data should be taken with care

\section{Results and Discussion}

The results of the SoS framework are presented in this section. The results provide answers to several research questions related to aerial wildfire fighting. These research questions span the multi-level framework System of Systems, system/aircraft and subsystem technology as shown in Fig. 12. The research questions are as follows:

1) Effect of number of Vehicles on Wildfire Suppression Success

2) Effect of Aircraft Cruise Speed on Wildfire Suppression Success

3) Effect of Response Time on Wildfire Suppression Success

4) Effect of Payload on Wildfire Burnt Area 


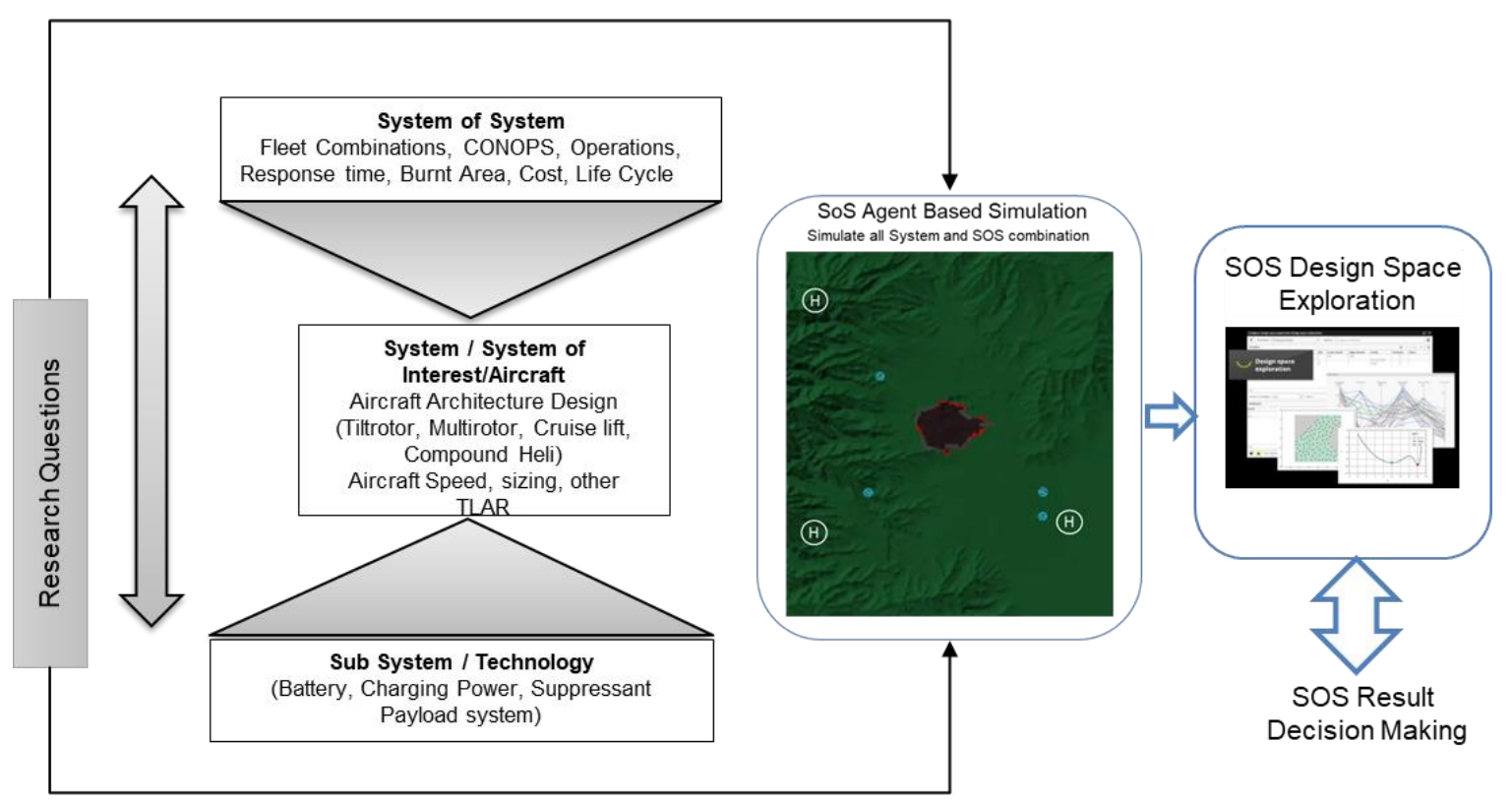

Fig. 12 System of System levels of the underlying research questions 


\section{A. Framework Capability Demonstration by Qualitative Results}

Before addressing the research questions in the following, the authors would like to introduce some qualitative results which demonstrates the capability of the framework. In Fig. 13 an active wildfire suppression mission is presented on the left with the fire represented by the red cells, whereas on the right is a successful suppression mission with the fire fully suppressed denoted by the grey cells.
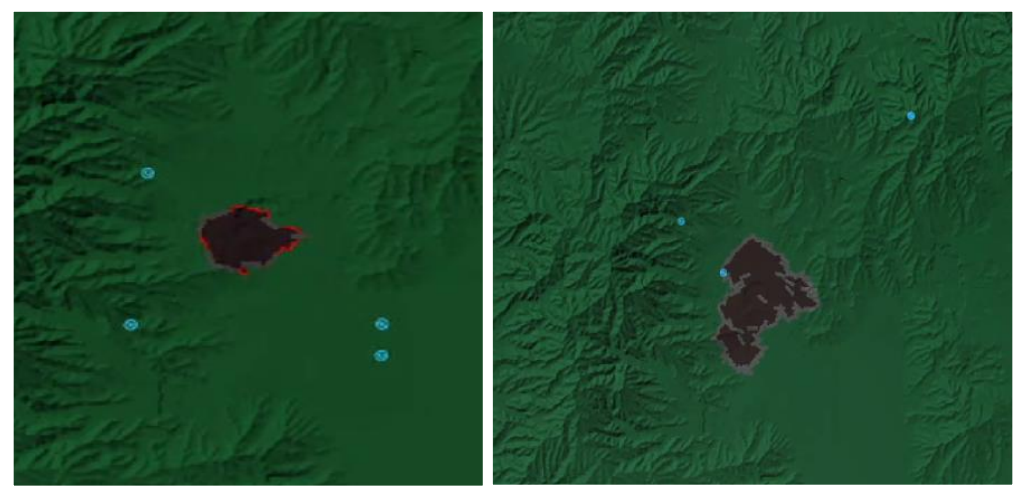

Fig. 13 Active wildfire suppression mission (left) and successful wildfire suppression mission (right)

The capability to prioritize certain locations is modeled in the wildfire suppression simulation. While in this study this capability is not utilized, it is presented here for completeness. On the left of Fig. 14, a failed wildfire suppression mission is presented with the protection locations (represented by yellow icons) disabled. The results of the simulation show that the agents naturally protect any location from which the water can be sourced. In this case these are UAV bases and off-base water sources. This behavior can be explained very easily. The default firefront selection is done based on the proximity to the agent. Since the agents are instructed to select the fire-front right after they have acquired the suppressant, that is at the UAV bases and off-base water sources, the fire-fronts closer to these locations will always have the priority. Consequently, the areas around water resupply locations are generally well protected. When the protection locations are enabled the wildfire suppression agents prioritize the area surrounding the protection locations, this can be seen to the right of Fig. 14. All firefighting capabilities are now used to stop the fire propagating towards the protection points. Only after these points are safe, the bases and water sources are defended. In the particular case displayed to the right of Fig. 14, one of the water sources was not spared from the flames. Because the fire reached this water source at the same time as one of the protection points was being threatened by an approaching fire-front, the protection point was given priority while the water source surroundings were left to burnt down. This capability was developed to model the priority areas such as towns which would be prioritized in a real-life suppression scenario.
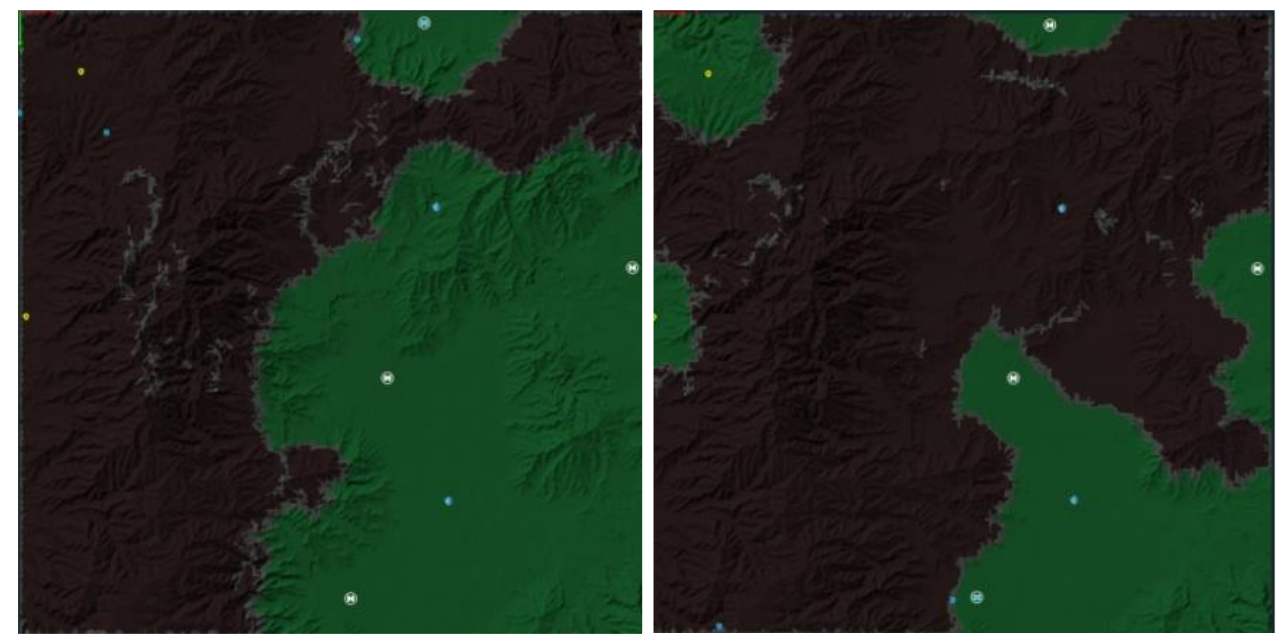

Fig. 14 Failed wildfire suppression missions with protection locations disabled (left) and protection locations enabled (right) 


\section{B. System of System Results for the Research Questions \\ 5. Effect of Number of Vehicles on Wildfire Suppression Success}

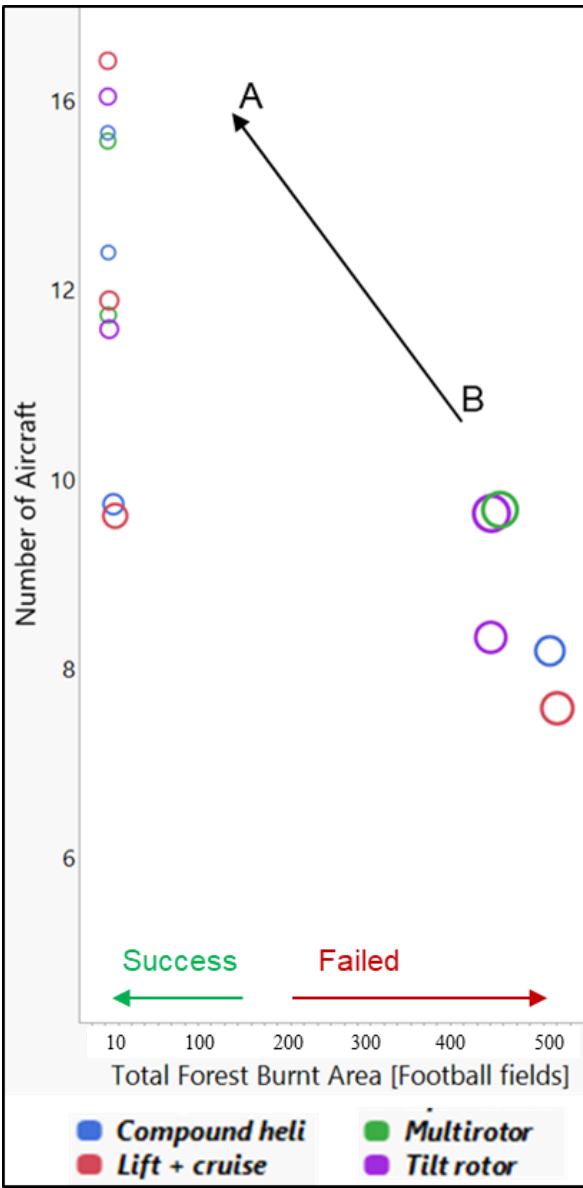

As shown in the Fig. 15, each circle represents a homogeneous fleet of the following aircraft configuration architectures :

Compound helicopter

Multirotor

Lift + Cruise

Tiltrotor

The units of measure of burnt area is given in terms of standard football fields for enhancing the comprehension of the reader. With 6 or 8 number of aircraft fighting the wildfire, there are no successful fleets of vehicles for any type of configuration. All of the fleet fail to suppress the fire or contain the wildfire.

With 10 vehicles of compound helicopter and lift + cruise configuration architecture the fire can be successfully contained, whereas the multirotor and tiltrotor configuration fail to suppress and there is a large burnt area of more than 450 football fields (the simulation stops after several hours as it will be impossible to contain the fire). This shows the configuration architecture effects of aircraft, i.e. system/system of interest level, impacted at System of System level.

With 12 or 16 vehicles, all configuration types can suppress the wildfire. The success trend is shown by Arrow AB.

The size of the circle represents the energy consumed by the aircraft. The larger circle represents more energy is required, highlighting more recharging sorties than suppression sorties

Fig. 15 Effect of aircraft numbers on wildfire suppression success(Short range, $250 \mathrm{~kg}$ payload)

\section{Effect of Aircraft Cruise Speed on Wildfire Suppression Success}

Fig. 16 highlights the effect of cruise speed $(20 \mathrm{~m} / \mathrm{s}, 30 \mathrm{~m} / \mathrm{s}, 40 \mathrm{~m} / \mathrm{s})$ on wildfire suppression. The boxed region "C" in Fig. 16 denotes that configurations have failed to suppress the fire while flying at $20 \mathrm{~m} / \mathrm{s}$. Box region "D" denotes that all type of vehicle configurations, i.e. compound helicopter (blue), lift + cruise (red), multirotor (green) and tiltrotor (purple), all flown at $30 \mathrm{~m} / \mathrm{s}$ successfully eliminate the fire within very few football fields of burnt area. Further, box region "E" denotes the cruise speed $40 \mathrm{~m} / \mathrm{s}$. Surprisingly, none of the vehicle configurations lead to successful missions. The reason being, flying faster needs higher energy and the aircraft spends more time recharging instead of fire suppressing sorties. Next figures show the actual magnitude of burnt area in successful missions. Thus, highlighting the diminishing effect of cruise speed. There is an optimal cruise for a successful SoS mission for each fleet composition. 


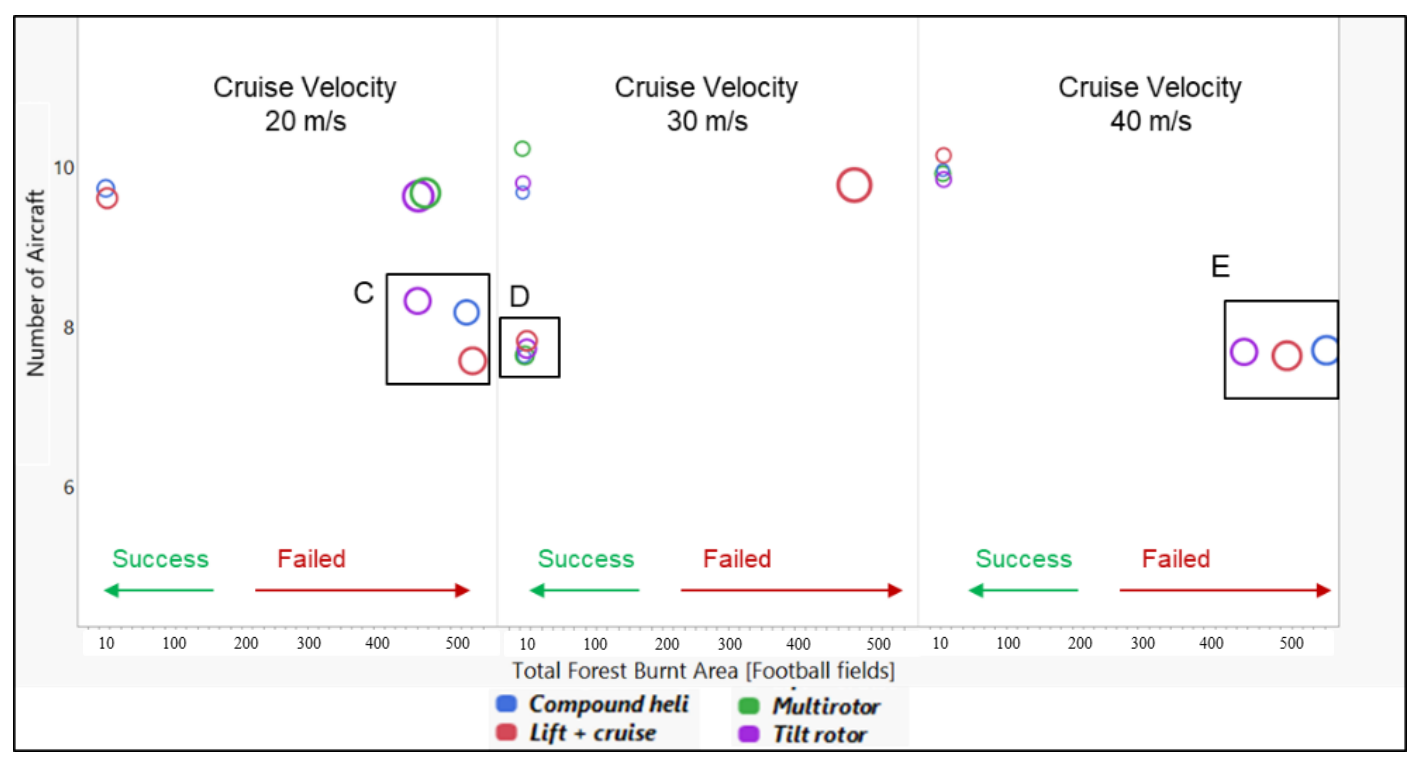

Fig. 16 Effect of cruise velocity on wildfire suppression success (Short range, $250 \mathrm{~kg}$ Payload)

Within the successful missions the effect of speed is shown in Fig. 17, where it can be noted that within the successful mission of the mid-range, $250 \mathrm{~kg}$ payload aircraft fleet, the configuration parameter play a role in how fast they can suppress fire. For example, if the homogenous fleet is flying at $20 \mathrm{~m} / \mathrm{s}$, the fleet with 12 aircraft can suppress the fire within 5 football fields as compared to 10 football fields with 10 aircraft. Adding just 2 aircraft can play a vital role in large burnt area reduction.

Also, the problem of diminishing returns (success) can be visualized at higher speeds and with higher number of aircraft. Such sensitivities or trends propagating aircraft/system level trends to System of System levels can be only assessed with a simulation embedded framework. 


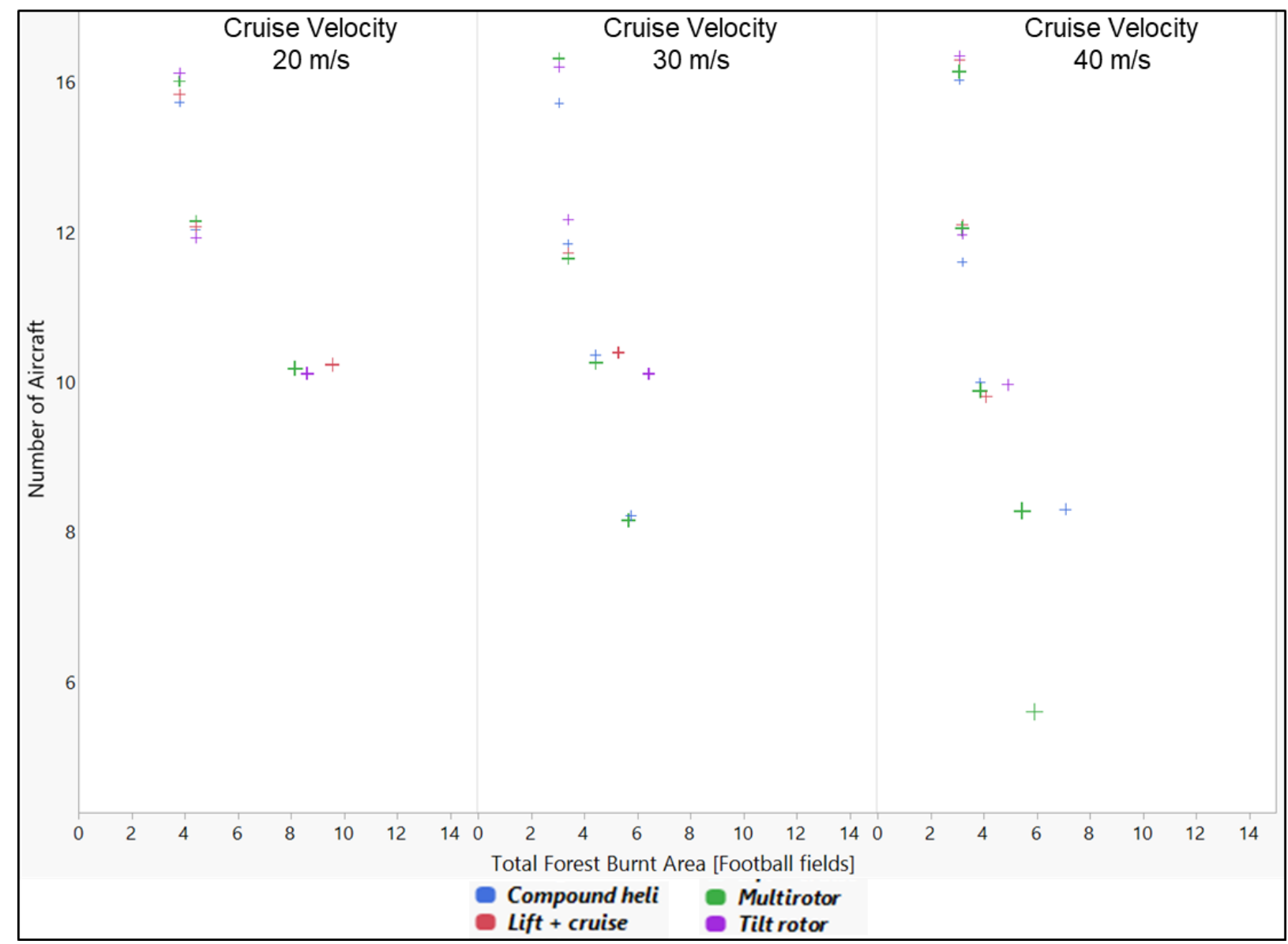

Fig. 17 Effect of cruise speed within successful missions (mid-range, $250 \mathrm{~kg}$ payload)

\section{Effect of Response Time on Wildfire Suppression Success}

Fig. 18 shows the effect of response time, the response time is defined as the time between the detection of fire and take off of the first wildfire fighting vehicle. When the response time increased by 5 minutes from 20 minutes to 25 minutes, as represented by box regions " $\mathrm{G}$ " and " $\mathrm{H}$ ", most configurations which was previously successful in suppression fails to suppress.

Thus, highlighting the fact that response time is important and SOS approach helps to evaluate such complex interactive phenomenon at fleet level. 


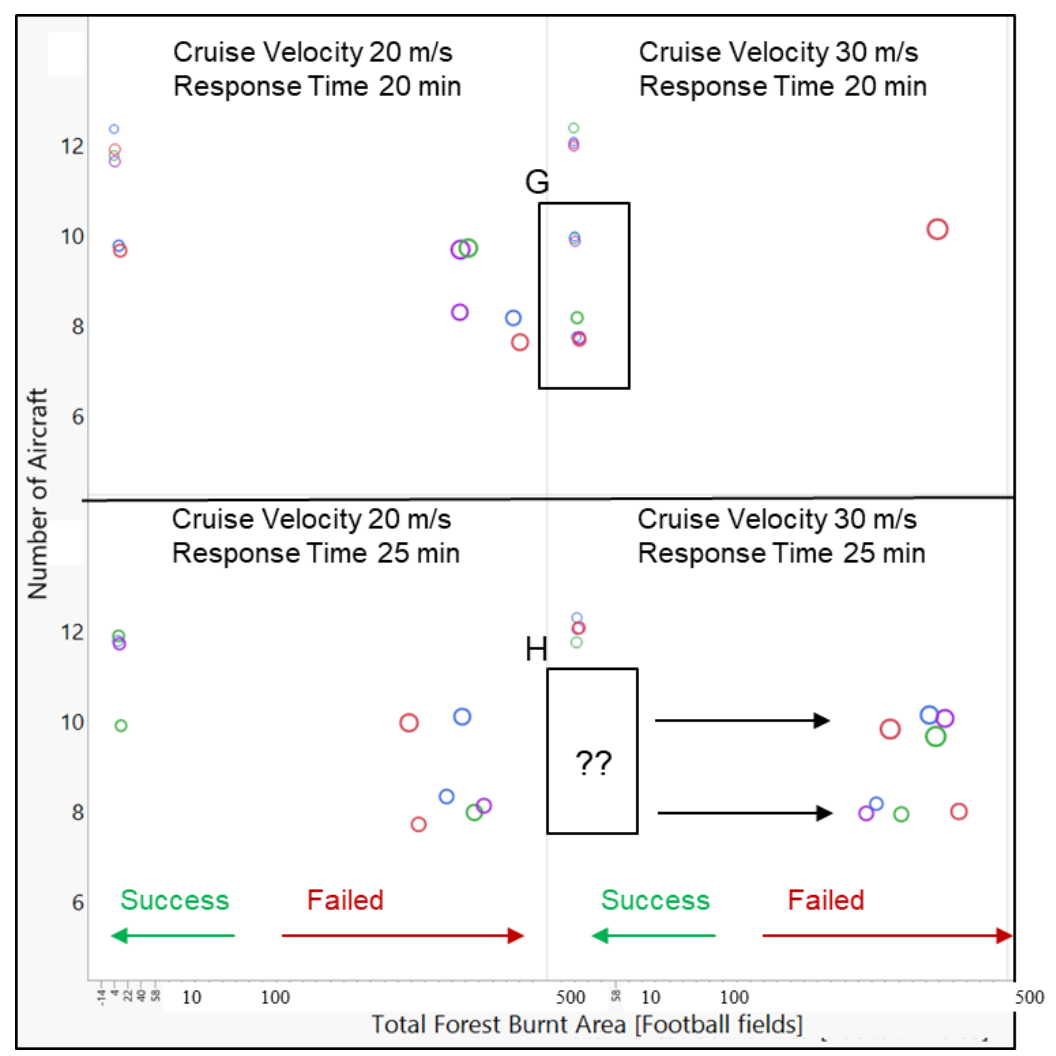

Fig. 18 Effect of response time on wildfire suppression success (short-range, $250 \mathrm{~kg}$ payload)

Within the successful missions, Fig. 19 shows the sensitivities of cruise speed, response time, and number of vehicles for different type of homogenous fleet. For example, $40 \mathrm{~m} / \mathrm{s}$ scenario of 25 minutes response time shows that $20 \%$ increase in fleet number from 10 to 12 would lead to $40 \%$ reduction in burnt area for tiltrotor (red) configuration.

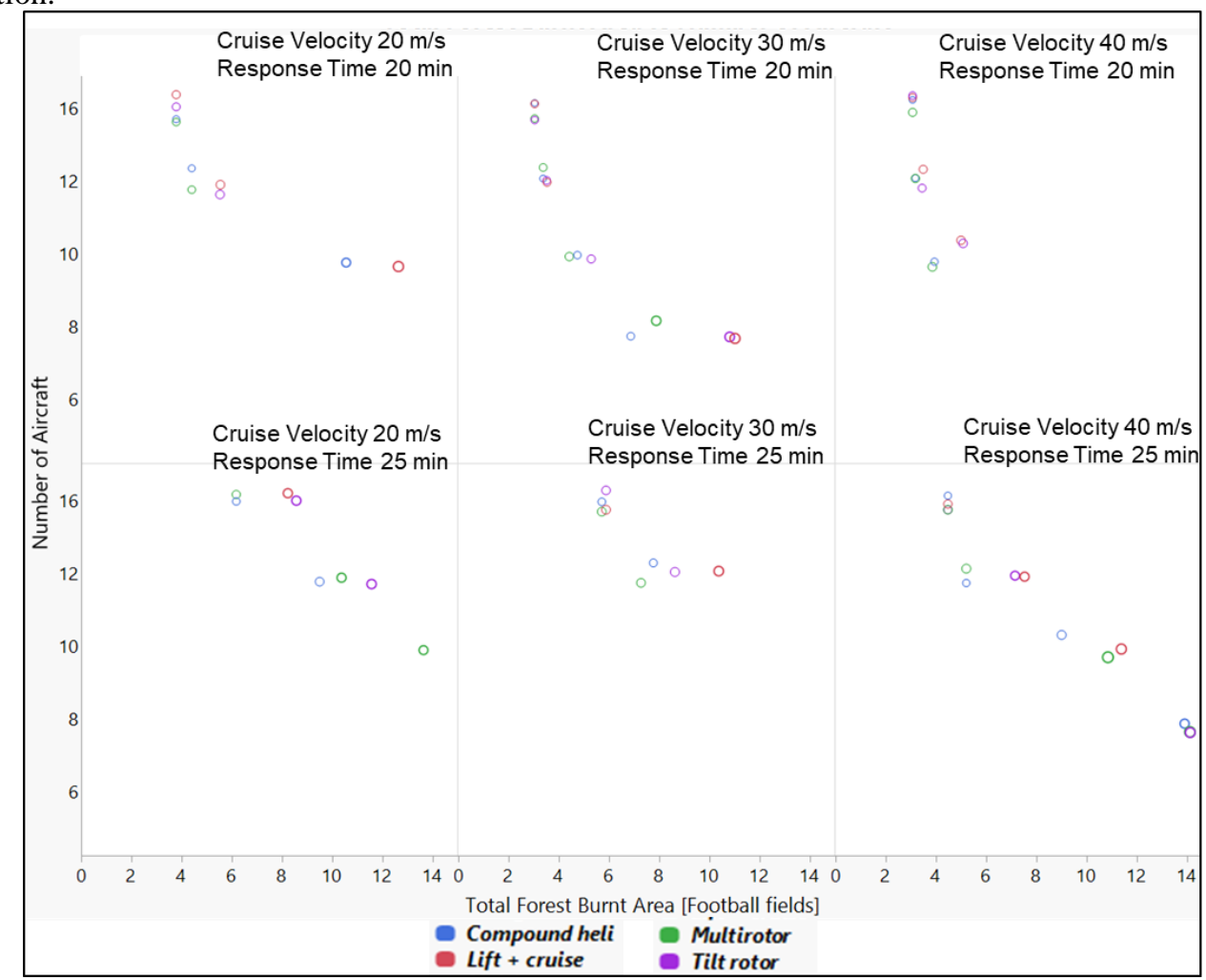

Fig. 19 Effect of response time within successful mission (short-range, $250 \mathrm{~kg}$ payload) 


\section{Effect of Payload on Wildfire Burnt Area}

Fig. 20 shows the effect of payload change from $250 \mathrm{~kg}$ to $500 \mathrm{~kg}$ of each aircraft. The performance of each aircraft is propagated based on weight (suppressant) carrying capability.

At $30 \mathrm{~m} / \mathrm{s}$ scenario, the multirotor (green) fleet can suppress the fire with 6 aircraft carrying $500 \mathrm{~kg}$ of payload or suppressant, but if it carries $250 \mathrm{~kg}$, the aircraft fail to suppress the fire. Similar trends can be seen for different fleet, speed, and weight combinations.

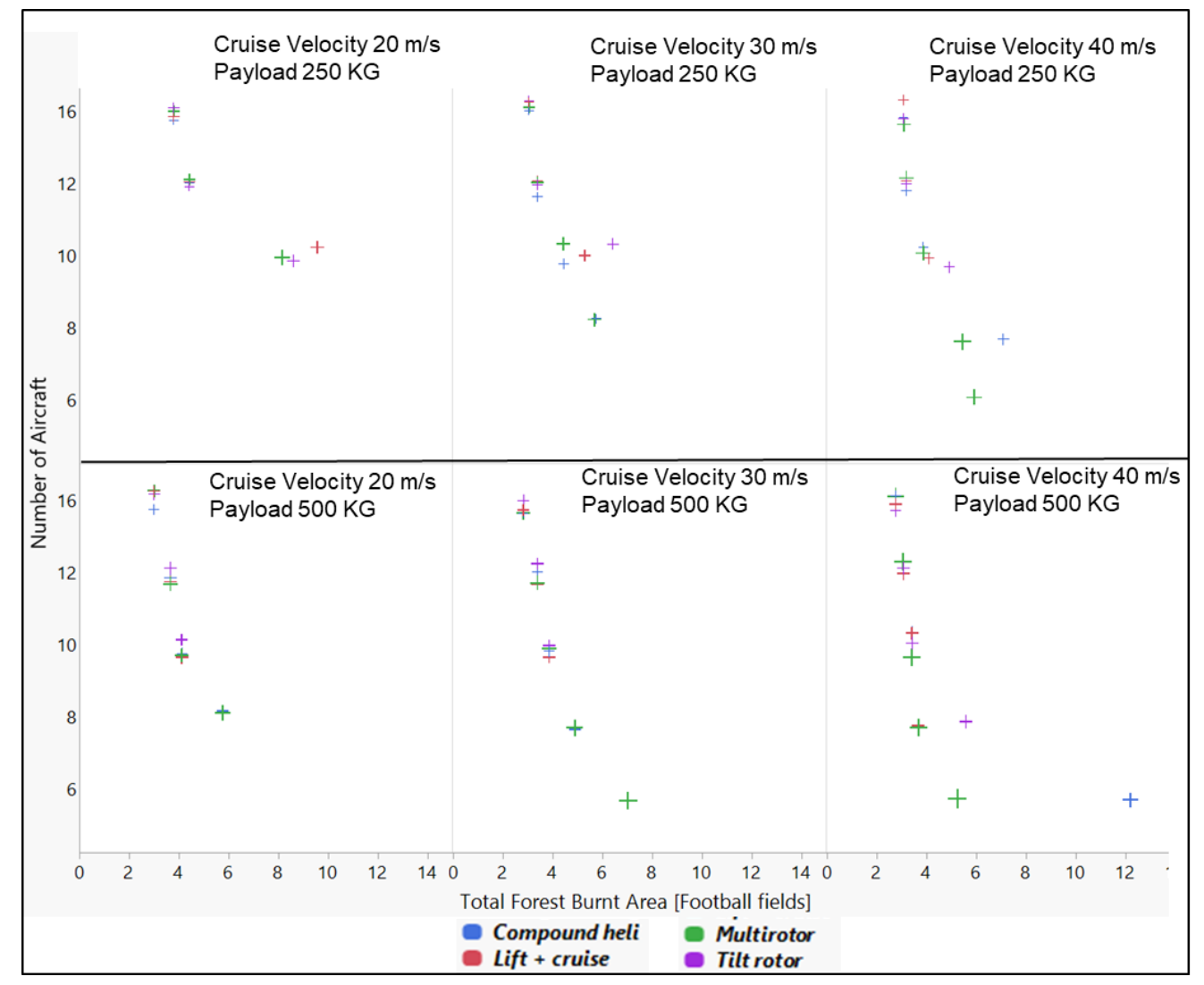

Fig. 20 Effect of payload on burnt area within successful missions (mid-range, 20 min response time)

\section{IV.Conclusion and Future Work}

The research questions, sensitivities and results highlight the fact that it is important to design the vehicles based on SOS approach to evaluate such complex interactive multi vehicle emergence phenomenon. Further supports narrowing down the design space for higher fidelity designs in next paper using the same framework. The focus of the study was to highlight sensitive parameters, technologies at vehicle level, and propagate it to fleet level and assess the fleet performance for fighting wildfire.

Within the simulation, the number of aircraft, fleet mix, base location and base numbers, water source are all parametrically modifiable. It should be noted that the framework can simulate various terrain, wind speed, vegetation burn rate, humidity, vegetation index, and wind direction. The demonstration of framework was limited to some constant parameters.

The effect of number of vehicles, payload, speed and design range has big impact on wildfire fighting success for a given scenario. A good combination of these properties (considered as fleet) will make or break the disaster response. Design of vehicle not to be performed as separate but with operations and heterogeneous or homogeneous fleet.

System of Systems driven design has non-linear couplings. For example, increasing cruise velocity would may not suppress fire faster, but may need more recharge sorties. There is an optimal for every technology, fleet and operation, beyond which the benefit diminishes or even hurt the optimum. Which makes analysis hard, simulations 
are time consuming but this is the only way to evaluate many nonlinear couplings of combinatorial evaluations to find the SoS emergence. This makes SoS and firefighting way more interesting.

Future work includes cost estimations as well as higher fidelity wildfire fighting UAV design, collaborative methods [14], heterogeneous fleets, and detailed vehicle and subsystem level performance estimations driven by other publications of same authors $[15,16]$. The technology, design, and methods uncertainties are propagated and quantified using the methods of Prakasha et al. [17].

\section{Acknowledgements}

The authors of this paper would like to express their gratitude to all the contributors of the SoSID toolkit and the past developers such as Miha Zupanic. Furthermore, additional thanks and gratitude are extended to the DLR Institute of System Architectures in Aeronautics for the possibility to conduct this research into System of Systems.

\section{Contact Author Email Address}

We would like to invite potential collaborators for this aerial firefighting project where we codevelop the firefighting simulation driven design. With access to existing simulation. Please contact author email address mailto: prajwal.prakasha@dlr.de or prajwal.prakasha@gmail.com 


\section{References}

[1] European Commission, "12. Climate action, environment, resource efficiency and raw materials," in Horizon 2020: Work Programme 2018-2020, 2019, p. 162.

[2] European Civil Protection and Humanitarian Aid Operations, European Union, and E. Scagnetti, "Emergency Response Coordination Centre (ERCC) Factsheet.” European Commission, 30-Jan-2019.

[3] M. W. Maier, "Architecting principles for systems-of-systems," p. 18.

[4] C. Yuan, Y. Zhang, and Z. Liu, "A Survey on Technologies for Automatic Forest Fire Monitoring, Detection and Fighting Using UAVs and Remote Sensing Techniques," Canadian Journal of Forest Research, vol. 45, p. 150312143318009,2015

[5] N. Castro, M. A. Akhloufi, and A. Couturier, "UAVs for wildland fires," in Autonomous Systems: Sensors, Vehicles, Security, and the Internet of Everything, Orlando, United States, 2018, p. 23.

[6] Bushfire Cooperative Research Center and Australian fire and Emergency Service Authorities Council, "Effectiveness and Efficiency of Aerial fire Fighting in Australia," Fire Note, no. 50, p. 4, Dec-2009.

[7] M. P. Plucinski, G. J. McCarthy, J. J. Hollis, and J. S. Gould, "The effect of aerial suppression on the containment time of Australian wildfires estimated by fire management personnel," Int. J. Wildland Fire, vol. 21, no. 3, p. 219, 2012.

[8] M. P. Plucinski, "Factors Affecting Containment Area and Time of Australian Forest Fires Featuring Aerial Suppression," Forest Science, vol. 58, no. 4, pp. 390-398, Aug. 2012.

[9] X. Rui, S. Hui, X. Yu, G. Zhang, and B. Wu, "Forest fire spread simulation algorithm based on cellular automata," Nat Hazards, vol. 91, no. 1, Art. no. 1, Mar. 2018, doi: 10.1007/s11069-017-3127-5

[10] P. Yang et al., "GSWO: A programming model for GPU-enabled parallelization of sliding window operations in image processing," Signal Processing: Image Communication, vol. 47, pp. 332-345, 2016, doi: https://doi.org/10.1016/j.image.2016.05.003

[11] Dominique Legendre et al. “Air tanker drop patterns". In: International Journal of Wildland Fire 23.2 (2014). DOI: 10.1071/WF13029, pp. 272-280.

[12] Brown, A. and Harris, W. L., "Vehicle Design and Optimization Model for Urban Air Mobility," Journal of Aircraft, vol. 57, no. 6, pp. 1003-1013, 2020. DOI: 10.2514/1.C035756.

[13] Kilkis, S., Naeem, N., Prakasha, P. S., Nagel, B., "A Python Modelling and Simulation Toolkit for Rapid Development of System of Systems Inverse Design (SoSID) Case Studies," AIAA Aviation 2021 Forum, 2021.

[14] Ciampa, P. D., Prakasha, P. S., Torrigiani, F., Walther, J.-N., Lefebvre, T., Bartoli, N., Timmermans, H. et al. "Streamlining Cross-Organizational Aircraft Development: Results from the AGILE Project," AIAA Aviation 2019 Forum, 2019 p. 3454. DOI: 10.2514/6.2019-3454

[15] Prakasha, P. S., Bertram, O., Ratei, P., Naeem, N., Nagel, B., "System of System Simulation driven Urban Air Mobility Vehicle Design," AIAA Aviation 2021 Forum, 2021.

[16] Prakasha, P. S., Boggero, L., Fioriti, M., Aigner, B., Ciampa, P. D., Anisimov, K., Isianov, A., "Collaborative system of systems multidisciplinary design optimization for civil aircraft: AGILE EU project," 18th AIAA/ISSMO Multidisciplinary Analysis and Optimization Conference, p. 4142, 2017

[17] Prakasha, P. S., Nam, T., Perullo, C. and Mavris, D. N., "Non Deterministic Approach for Advanced Aircraft Configuration Design under Uncertainty," 15th AIAA/ISSMO Multidisciplinary Analysis and Optimization Conference, p. 2182, 2014. 